\title{
The two domains of cotton WLIM1a protein are functionally divergent
}

\author{
Libo $\mathrm{Han}^{1 *}$, Yuanbao $\mathrm{Li}^{1,2}$, Yongduo Sun ${ }^{1,2}$, Haiyun $\mathrm{Wang}^{1}$, Zhaosheng Kong ${ }^{1}$ \& Guixian Xia ${ }^{1}$ \\ ${ }^{1}$ State Key Laboratory of Plant Genomics, Institute of Microbiology, Chinese Academy of Sciences, Beijing 100101, China; \\ ${ }^{2}$ College of Life Sciences, University of Chinese Academy of Sciences, Beijing 100049, China
}

Received May 14, 2015; accepted July 20, 2015; published online January 22, 2016

\begin{abstract}
Our previous study demonstrated that WLIM1a has dual roles in fiber elongation and secondary cell wall synthesis in upland cotton, and the protein acts either as an actin-binding protein or as a transcription factor. Because WLIM1a consists of two different LIM domains, it is possible that these elements contribute differentially to the dual functions of the protein. In this study, we dissected the two LIM domains and characterized their biochemical functions. By using red fluorescent protein (RFP) fusion, co-sedimentation, and DNA binding methods, we found that the two domains of WLIM1a, domain1 (D1) and domain2 (D2), possessed different biochemical properties. While D1 contributed primarily to the actin filament-bundling activity of WLIM1a, D2 contributed to the DNA-binding activity of the protein; both D1 and D2 relied on a linker sequence for their activities. In addition, we found that WLIM1a and its two LIM domains form dimers in vitro. These results may lead to a better understanding of the molecular mechanisms of dual functions of WLIM1a during cotton fiber development.
\end{abstract}

WLIM1a, actin bundle, transcription factor, dual function

Citation: Han, L., Li, Y., Sun, Y., Wang, H., Kong, Z., and Xia, G. (2016). The two domains of cotton WLIM1a protein are functionally divergent. Sci China Life Sci 59, 206-212. doi: 10.1007/s11427-016-5002-0

\section{INTRODUCTION}

Cotton (Gossypium hirsutum) fiber develops from a single epidermal cell of the seed coat. Fiber development can be divided into four overlapping stages: initiation, elongation, secondary wall deposition, and maturation (Graves and Stewart, 1988). Growth and development in these stages determine fiber properties. Particularly, fiber elongation determines length while secondary wall synthesis determines traits of fineness and strength. Owing to its exceptional cell length and simple secondary cell wall composition, cotton fiber provides an excellent model for studies of plant cell elongation and cell wall biogenesis (Kim and Triplett, 2001). Recently, significant progress has been made in large-scale identification of genes and proteins in-

*Corresponding author (email: hanlibo@im.ac.cn) volved in fiber development, particularly of those related to fiber elongation and secondary wall deposition (Arpat et al., 2004; Cao, 2015; Gou et al., 2007; Jin et al., 2013; Shi et al., 2006; Zhao et al., 2010). Moreover, a number of fiber development-related genes have been structurally or functionally characterized (Han et al., 2013; Li et al., 2005; Pei, 2015; Ruan et al., 2003; Shan et al., 2014; Xu et al., 2013; Zhang et al., 2011). These studies have made important progress towards understanding the molecular mechanisms governing cotton fiber development.

LIM proteins are named for the initials of three homeodomain proteins (LIN-11, ISL1, and MEC-3). These proteins were reported to act as developmental regulators in eukaryotes, participating in a variety of basic cellular processes including gene transcription, cytoskeletal organization, and signaling (Dawid et al., 1995). The LIM domain, with the consensus sequence [C-X2-C-X16-23-H-X2-C]- 
$\mathrm{X} 2-[\mathrm{C}-\mathrm{X} 2-\mathrm{C}-\mathrm{X} 16-21-\mathrm{C}-\mathrm{X} 2-3-(\mathrm{C} / \mathrm{D} / \mathrm{H})]$ that essentially comprises two zinc fingers linked together by a short 2-amino acid spacer, functions as a protein-protein interaction module (Zheng and Zhao, 2007). Compared with animal genomes that contain multiple LIM genes, plants genomes encode a limited number of LIM genes. Plant LIM proteins have been reported to function in either actin binding to promote formation of actin bundles or in DNA binding to regulate gene expression (Han et al., 2013; Moes et al., 2013; Zheng and Zhao, 2007). Tobacco WLIM1 and WLIM2 and six Arabidopsis LIM proteins are vital to bundle actin filaments and protect the actin cytoskeleton from disassembly (Dawid et al., 1995; Zheng and Zhao, 2007). In addition, they are key trans-acting factors regulating expression of genes involved in phenylpropanoid biosynthesis and cell division (Kawaoka et al., 2000; Thomas et al., 2006). Our previous work has shown that the cotton LIM domain protein, WLIM1a, has dual roles during fiber development (Han et al., 2013). WLIM1a may act as an actin bundler to promote cell elongation at the fiber-cell elongation stage or as a transcriptional factor to trigger lignin biosynthesis at the secondary cell wall biosynthesis stage. WLIM1a can bind both actin filaments and DNA, localizes in the cytosol and nucleus, and moves into the nucleus in response to hydrogen peroxide (Han et al., 2013).

The WLIM1a protein has two LIM domains and can execute two distinct biochemical functions; this fact caused us to investigate whether these functions of WLIM1a depend on different structural domains of the protein. To address this question, in this study, we characterized the biochemical functions of the two domains of WLIM1a in vitro. Results from high-speed and low-speed cosedimentation, microscopy, and electrophoretic mobility shift assay (EMSA) show that LIM domain1 (D1) contributed primarily to actin bundling and LIM domain2 (D2) contributed mainly to DNA binding. Our findings may help elucidate molecular mechanisms by which WLIM1a executes dual functions in cotton fiber development.

\section{RESULTS}

\section{WLIM1a contains two LIM domains with low sequence similarity}

The cotton WLIM1a protein is a 190-amino acid protein that comprises of a short $\mathrm{N}$-terminal domain $(\mathrm{Nt}, 9$ amino acids), two typical LIM domains (D1 and D2; 56 amino acids each) separated by a 44-amino acid linker sequence L, and a C-terminal domain ( $\mathrm{Ct}, 25$ amino acids) (Figure 1A). Sequence alignment (Figure 1B) shows that WLIM1a shares high sequence similarity with other plant LIM- domain proteins such as AtWLIM1, AtPLIM2c (Papuga et al., 2010), LlLIM1 (Wang et al., 2008), Ntlim1 (Kawaoka et al., 2000), and NtWLIM1 (Thomas et al., 2006). Interestingly, the two LIM domains of WLIM1a only share $40 \%$

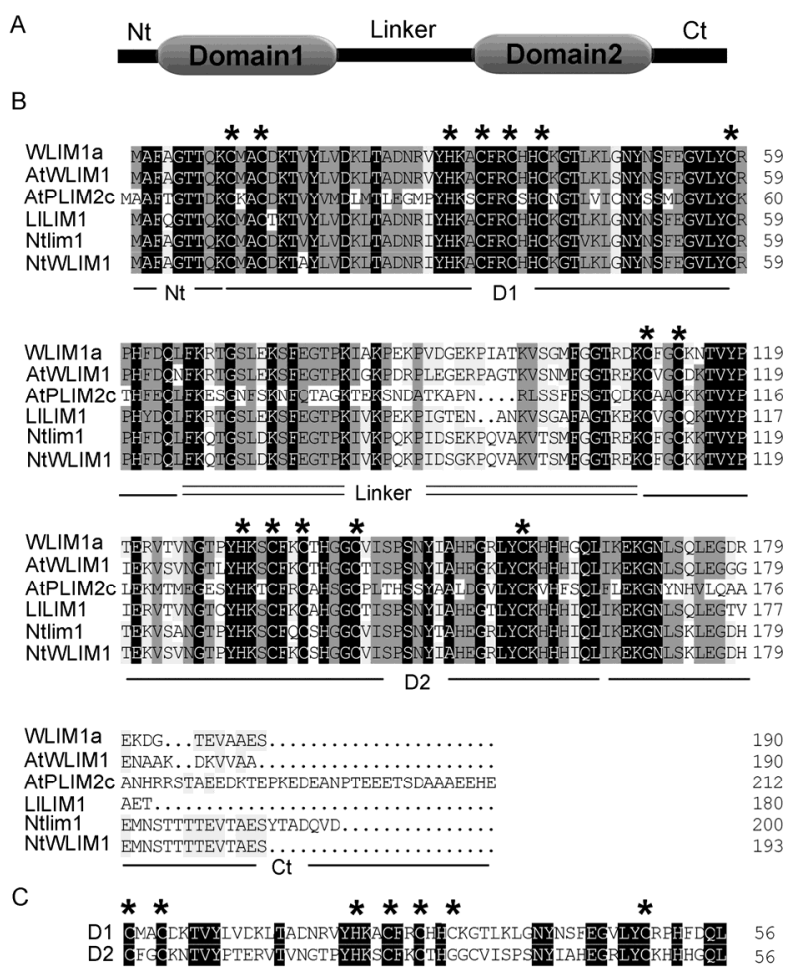

Figure 1 Motif structures of cotton WLIM1a, and alignment of amino acid sequences of plant LIM-domain proteins. A, Schematic of motif structures of the WLIM1a protein. B, Amino acid sequence alignment of WLIM1a with other plant LIM-domain proteins. Black shading, identical and conserved amino acids; gray shading, similar amino acids; asterisks, identical Cys or His residues in LIM domains. C, Sequence alignment of the two LIM domains of cotton WLIM1a. Black shading, identical amino acids between the two LIM domains; asterisks, identical Cys or His residues in LIM domains.

amino acid sequence identity although both contain a 56-amino acid LIM motif (Figure 1C). The low sequence identity of D1 and D2 suggests that they may have different functional properties.

\section{Generation of $6 \times$ His-tagged recombinant WLIM1a and WLIM1a variants}

In our previous study, we found that WLIM1a could bind to filamentous actin (F-actin) and to a PAL-box DNA element (Han et al., 2013). Binding of WLIM1a to these two targets raised the question of whether these distinct functions of WLIM1a depend on different structural domains of the protein. To address this question, we generated five protein variants (D1L, LD2, D1, D2, and L) of WLIM1a, and the derived peptides are shown in Figure 2A. The recombinant proteins were expressed in Escherichia coli with a $6 \times$ His tag at the $\mathrm{C}$ terminus, and purified on a nickel-nitrilotriacetate-agarose matrix (Figure 2B). As shown in Figure 2B, the full length WLIM1a and protein variants were successfully expressed and purified. These proteins were used in subsequent in vitro experiments. 


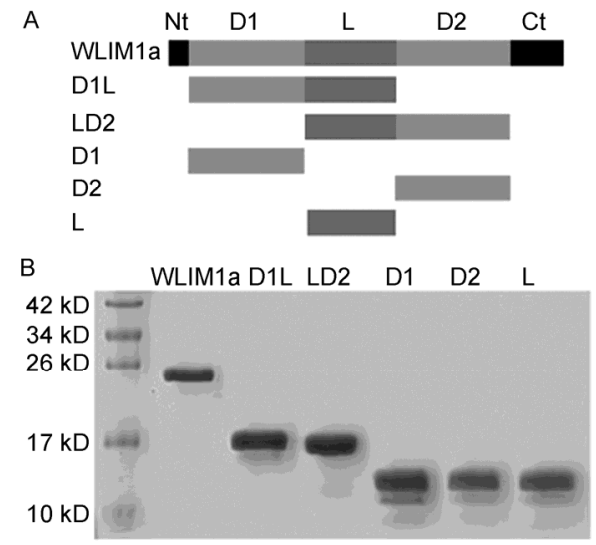

Figure 2 Motif composition and production of WLIM1a and its truncated variants. A, Diagram of motif composition of the WLIM1a protein. D1, domain1; D2, domain2; L, the inter-LIM linker sequence. B, Production of intact and truncated LIM proteins in Escherichia coli. Molecular masses of standard proteins are indicated on the left.

\section{D1 contributes primarily to the actin filament-bundling activity of WLIM1a}

The ability of each protein portion to bind to F-actin was assayed in high-speed $(200,000 \times g)$ cosedimentation assays. Proteins were incubated for $1 \mathrm{~h}$ with polymerized F-actin, the mixture was centrifuged, and the resulting pellet and supernatant fractions were analyzed by sodium dodecyl sulfate polyacrylamide gel electropheresis (SDS-PAGE). As shown in Figure 3A, WLIM1a, D1L, and LD2 were enriched in the pellet fraction together with F-actin, and a small amount of D1 and D2 was present in the pellet fraction. In contrast, peptide $\mathrm{L}$ was detected only in the supernatant fraction. These data demonstrate that WLIM1a, D1L, LD2, D1, and D2 can interact directly with F-actin in vitro except for the linker peptide $\mathrm{L}$.

Low-speed cosedimentation was used to determine F-actin bundling properties of these truncated proteins. Actin $\left(3 \mu \mathrm{mol} \mathrm{L}{ }^{-1}\right)$ was polymerized alone or in the presence of $3 \mu \mathrm{mol} \mathrm{L}{ }^{-1}$ native WLIM1a or truncated variants and centrifuged for $30 \mathrm{~min}$ at $13,500 \times \mathrm{g}$. Both supernatant and pellet fractions were analyzed by SDS-PAGE. As shown in Figure 3B, in the absence of native WLIM1a, traces of actin aggregates were detected in the pellet fraction. Moreover, when LIM proteins were added to polymerized F-actin samples, addition of native WLIM1a, D1L and LD2 increased the amount of actin in the pellet fraction. These results suggest that intact WLIM1a, D1L, and LD2 can promote actin filaments bundling, but D1 and D2, which lack the linker sequence, can not bundle actin. Compared to native WLIM1a and D1L, LD2 had weak actin-bundling activity.

To further confirm the actin binding/bundling activity of these LIM proteins, we fused the LIM sequences to the flu-

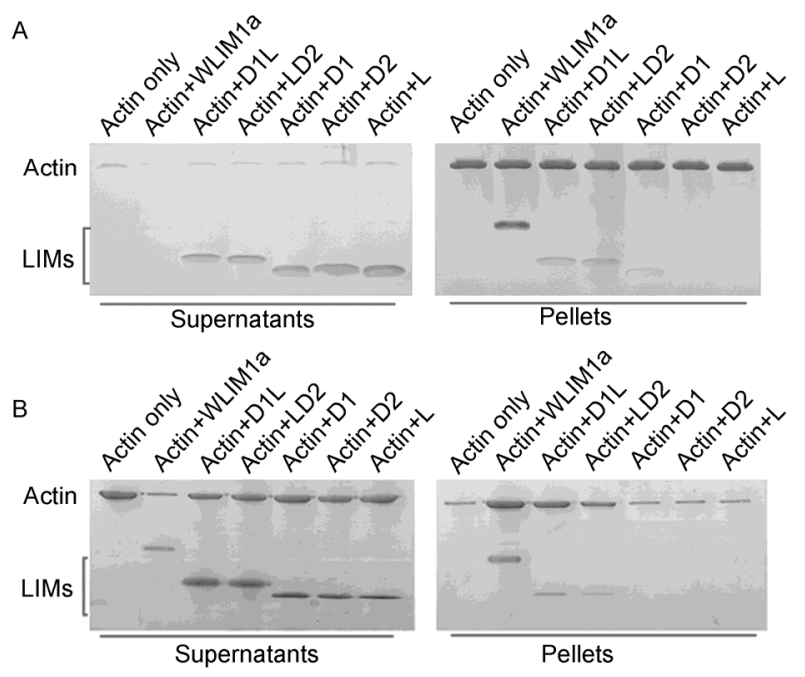

Figure 3 F-actin binding/bundling activity of WLIM1a and truncated LIM proteins. A, High-speed cosedimentation assay. Proteins in the supernatant and pellet fractions were analyzed by SDS-PAGE. B, Low-speed cosedimentation assay. Proteins in the supernatant and pellet fractions were analyzed by SDS-PAGE.

orescent marker RFP to generate WLIM1a-RFP, D1L-RFP, LD2-RFP, D1-RFP, D2-RFP, and L-RFP recombinant proteins. We then incubated these recombinant proteins with preassembled F-actin, and the F-actin binding/bundling activity of these proteins was confirmed by visualizing actin filaments stained with Alexa Fluor 488 phalloidin. Figure $4 \mathrm{~A}$ and $\mathrm{B}$ show that the actin filaments were decorated by WLIM1a-RFP, D1L-RFP, and LD2-RFP proteins. Some D1-RFP and D2-RFP protein spots localized at the actin filaments; the L-RFP signal did not colocalize with actin filaments. These observations indicate that all LIM protein variants, except $\mathrm{L}$, could bind to actin filaments. In addition, we noticed that only WLIM1a-RFP, D1L-RFP, and LD2-RFP could bundle actin filaments; D1 and D2 do not possess this F-actin bundling property. Compared with WLIM1a-RFP and D1L-RFP, LD2-RFP induces less F-actin bundling.

\section{D2 mainly contributes to the PAL-box DNA-binding activity of WLIM1a}

EMSA was performed to investigate the PAL-box DNA binding activity of His-tagged LIM proteins. A retarded band showing DNA binding activity of native WLIM1a protein was observed (Figure 5, lane 2). Moreover, we found that retarded bands appeared when D1L and LD2 were incubated with PAL-box DNA probes and the amount of the binding DNA probes decreased in accordance with the decreased amount of D1L and LD2 proteins (Figure 5, lane 3-8). Furthermore, LD2 bound more strongly to DNA probes compared to D1L at the same experimental conditions (Figure 5, lane 3 and 6). In addition, D1, D2, and L alone were tested for binding to the PAL-box. The results showed that they could not promote formation of any re- 

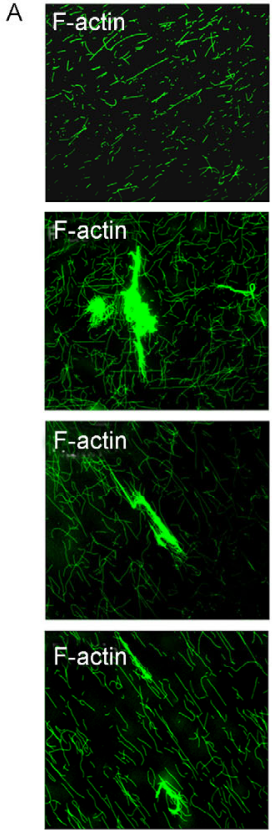
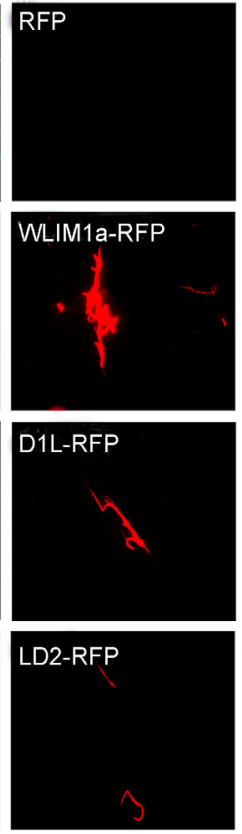
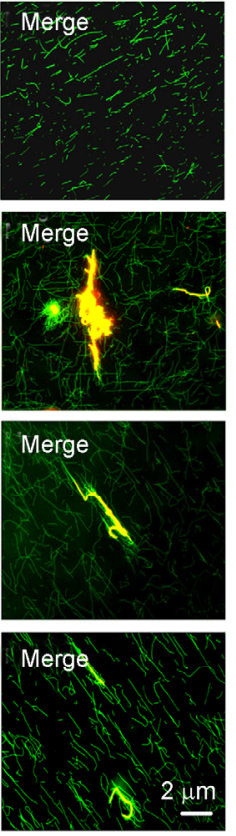
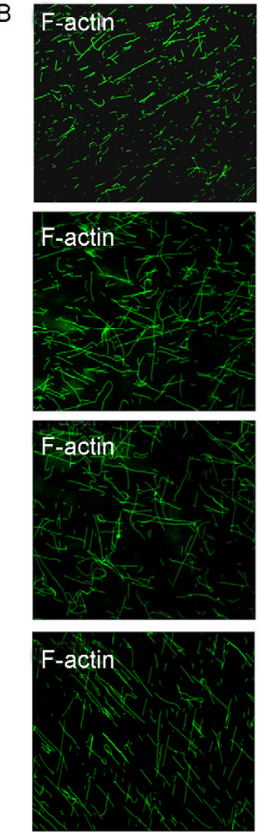
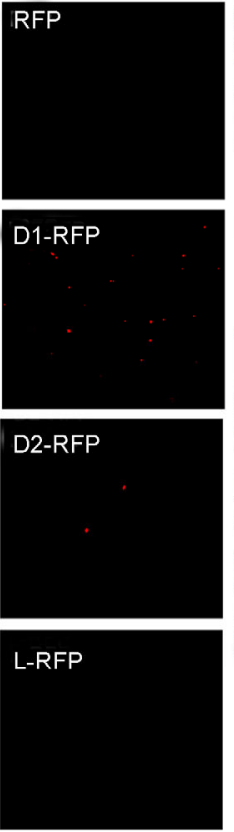
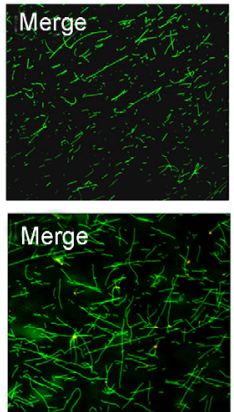

Merge
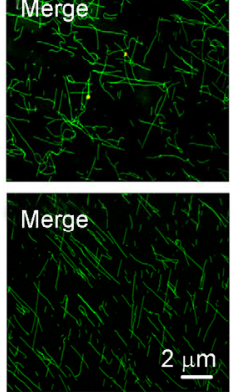

Figure 4 Fluorescence microscopy analysis of F-actin binding/bundling activities of WLIM1a and truncated LIM proteins. A, F-actin binding/bundling activities of His-tagged LIM-RFP proteins (WLIM1a, D1L, LD2, and RFP). RFP was used as a negative control. B, F-actin binding/bundling activities of His-tagged LIM-RFP proteins (D1, D2, and L). RFP was used as a negative control.

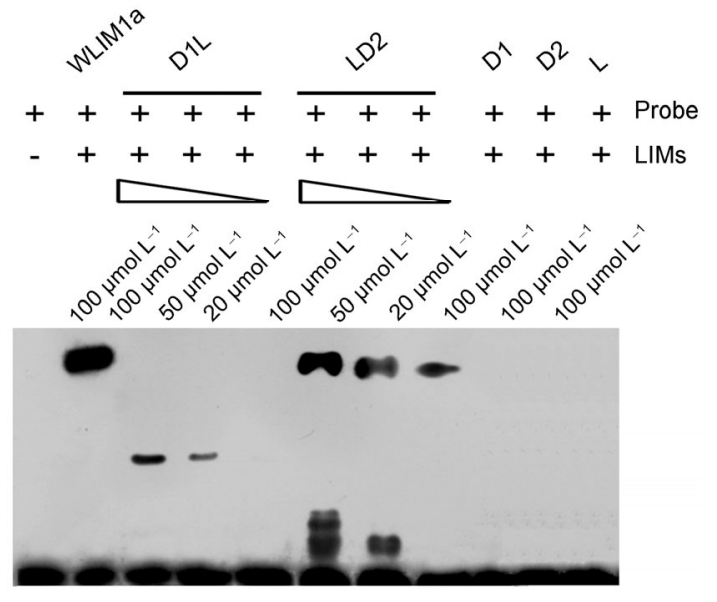

Figure 5 PAL-box binding activity of WLIM1a and truncated LIM proteins. Biotin-labeled PAL-box DNA fragments, probes alone (lane 1) or incubated with indicated His-tagged WLIM1a or the truncated LIM proteins (lane 2-11), were subjected to the gel shift assay.

tarded bands, indicating that the three peptides (D1, D2, and L) did not bind DNA probes (Figure 5, lane 9-11). In summary, we investigated the actin binding/bundling and PAL-box DNA binding activities of the LIM variants. As shown in Table 1, native WLIM1a and truncated D1L, LD2, D1, and D2 cosedimented and colocalized with F-actin, indicating that these segments are capable of binding to actin filaments. However, low-speed cosedimentation assays and microscopic visualization of actin filaments revealed a disparity in actin-bundling activities of these peptides. Native WLIM1a, D1L, and LD2 promoted actin fila-
Table 1 Functions of WLIM1a and truncated LIM proteins ${ }^{\text {a) }}$

\begin{tabular}{ccc}
\hline Proteins & F-actin bundling activity & PAL-box binding activity \\
\hline WLIM1a & +++++ & +++++ \\
D1L & +++ & ++ \\
LD2 & + & ++++ \\
D1 & - & - \\
D2 & - & - \\
L & - & - \\
\hline
\end{tabular}

a) Above summary is based on results depicted in Figures 3, 4, and 5 . "+" indicates the activity of the protein; multiple "+" indicate higher activity of the protein in F-actin bundling or DNA binding; "-" indicates no activity.

ment bundling, but D1 and D2, which lacked the linker sequence $\mathrm{L}$, did not bundle actin, indicating that the linker sequence is required for actin bundling functions. Compared to D1L, LD2 had weak actin-bundling ability. Based on these data, we can rank F-actin bundling activities of the three peptides as follows: WLIM1a $>$ D1L $>$ LD2. The order of D1L and LD2 was inversed when their PAL-box binding properties were compared: LD2 bound more strongly to the DNA probes compared to D1L. D1, D2, and the linker L alone did not bind to the PAL-box. Thus, PAL-box binding activities of intact WLIM1a and truncated proteins were ranked in the following order: WLIM1a $>$ LD2 $>$ D1L.

Together, these results indicate that the dual functions of WLIM1a can be attributed to the two LIM domains, with D1 contributing primarily to actin bundling and D2 contributing mainly to PAL-box binding. In addition, both D1 and D2 relied on the linker sequence for their activities. 
Interestingly, we found that LD2 and PAL-box formed a complex with much lower mobility compared to the D1LPAL-box complex. This highly reproducible result reflected the structural dissimilarity of the two domains.

\section{WLIM1a and its truncated proteins can form dimers in solution}

It has been reported that LIM proteins form dimers that facilitate actin bundling (Thomas et al., 2007). To assess if WLIM1a and its LIM domains can form dimers, we expressed GST-WLIM1a, GST-D1L, GST-LD2, GST-D1, GST-D2, and GST-L in E. coli. Figure 6A shows the purified GST-LIMs. GST fusion proteins bound to glutathione beads were used as bait and incubated with His-tagged LIM proteins. As shown in Figure 6B, except peptide L, all LIM proteins could be pulled down by themselves indicating that they could form homodimers. Alternatively, we also used the His-tagged LIM proteins as baits and incubated with GST-tagged LIM proteins. The results showed that LIM domain proteins possess the property of self-interaction or could form dimers (Figure 6C).

\section{DISCUSSION}

LIM-domain proteins have been identified in several plants. In Arabidopsis, all six LIM-domain proteins were found to decorate actin filaments (Papuga et al., 2010). In tobacco,

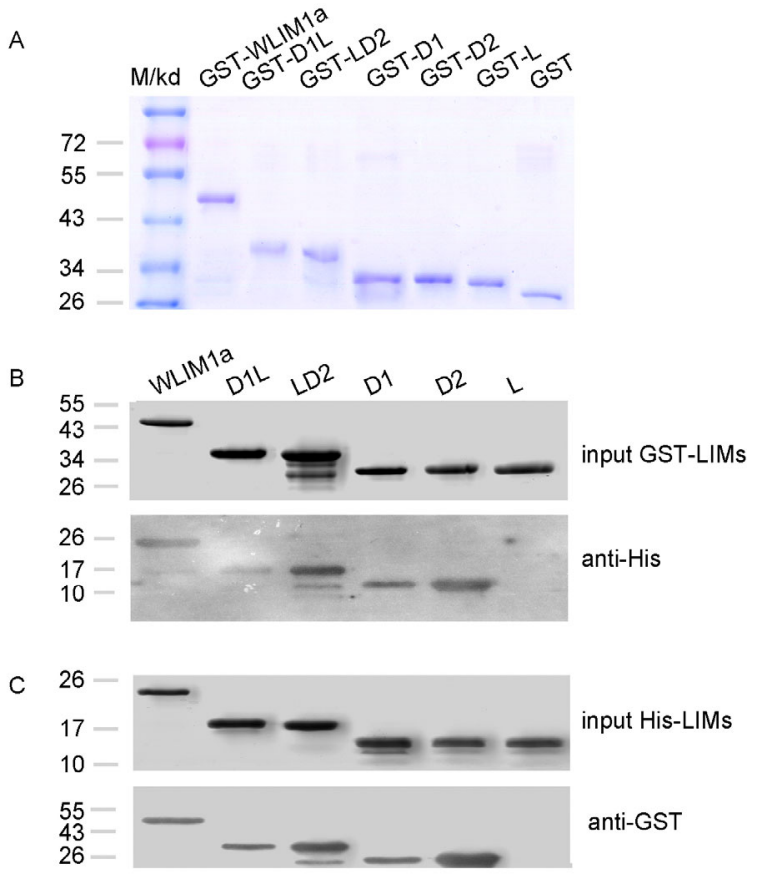

Figure 6 Dimerization analysis of WLIM1a and truncated LIM proteins. A, SDS-PAGE analysis of purified proteins. GST-WLIM1a and truncated GST-LIM proteins were expressed in Escherichia coli, and the purified proteins were subjected to SDS-PAGE. Molecular masses of standard proteins are indicated on the left. B and C, Pull-down assay showing dimerization of WLIM1a and truncated LIM proteins.
NtWLIM1 and NtWLIM2 were found to be both actin binding/bundling proteins and transcriptional regulators that activate the expression of prxC2 and histone, respectively (Kawaoka and Ebinuma, 2001; Moes et al., 2013). We recently reported the characterization of a dual functional LIM protein from upland cotton, namely the cotton WLIM1a protein. This LIM domain-containing protein was shown to interact directly with actin filaments and bundle them in vitro and in plant cells to facilitate elongation of fiber cells; in addition, this protein can bind to the PAL-box DNA motif to activate expression of PAL-box genes involved in phenylpropanoid biosynthesis to synthesize the secondary cell wall (Han et al., 2013). Together, these studies provide solid evidence demonstrating the dual functions of plant LIM-domain proteins.

The dual activities of WLIM1a caused speculation over the mechanism by which a single small protein could exert two distinct functions. Because the WLIM1a protein contains two LIM domains, we investigated whether there is a functional divergence between these two motifs. By domain dissection, we found that the LIM domain2 had a much higher affinity to the PAL-box sequence compared to LIM domain1. In contrast, the actin-bundling assay showed that LIM domain1 has a stronger actin-bundling activity compared to LIM domain2. In addition, EMSA assay showed that the linker sequence of WLIM1a was essential for the PAL-box-binding activity of domain 2 and for the actin bundling activity of domain 1 . Thus, by virtue of their distinct abilities, the two individual LIM domains and the linker region contribute differently to the two functions of WLIM1a. Consistent with our data, the LIM domain1 of tobacco WLIM1, was shown to be more efficient in actin bundling compared to the LIM domain2 of NtWLIM1 (Thomas et al., 2007), and both LIM domain1 and LIM domain2 of NtWLIM1 were found to be sufficient for the DNA binding activity of the protein (Kawaoka et al., 2000). It should be stressed that in our experiments, we furthermore observed that the actin-bundling activity of LIM domain1 and the PAL-box-binding activity of LIM domain2 were weaker than those of the native, non-truncated protein. This result indicates that although the two domains of WLIM1a are functionally divergent, they are not completely independent. The two LIM domains of WLIM1a share $40 \%$ amino acid sequence identity; therefore, it is possible that the WLIM1a protein was assembled by a genomic rearrangement that combined two proteins with distinct functions. Alternatively, LIM domain1 and LIM domain2 may originate from the same ancestral protein and their individual functions diverged later, causing functional diversification.

It is known that the plant actin-binding proteins such as villin and formin require dimerization for their actin bundling activity (George et al., 2007; Xue et al., 2011). Concerning LIM proteins, one assumption is that LIM domains could dimerize to fulfill their actin-bundling function 
(Thomas et al., 2007). Combined with our results, which suggest that both LIM domains directly bundle actin filaments (Figures 3 and 4) and truncated WLIM1a peptides are capable of forming dimers as verified by pull-down assay (Figure 6), we hypothesize that neighboring LIMs bundle actin filaments through dimerization. Interestingly, we further observed that D2 could form dimers in vitro, suggesting that dimerization may also be needed for the DNA binding activity of WLIM1a. Further study is required to assess these possibilities.

\section{MATERIALS AND METHODS}

\section{Alignment of the amino acid sequence of WLIM1a with that of other LIM domain protiens}

Protein sequences of WLIM1a and other previously reported LIM domain proteins (AtWLIM1, AtPLIM2c, LILIM1, Ntlim1, and NtWLIM1) were aligned using the ClustalW software.

\section{Expression and analysis of recombinant proteins}

The opening reading frame (ORF) and cDNA sequences for the WLIM1a domains (LIM domain1 (aa10-65, D1), domain1+linker (aa10-109, D1L), LIM domain2 (aa110-165, D2), linker+domain2 (aa66-165, LD2), and linker (aa66$109, \mathrm{~L})$ ) were cloned into the bacterial expression vector pET28a or pGEX 6P-1 vector and expressed in Escherichia coli strain BL21 (DE3). His-tagged recombinant intact or truncated LIM proteins were purified using Ni-NTA resin by following procedures described by the manufacturer (Qiagen, Germany), and the GST-tagged recombinant proteins were purified using glutathione-sepharose 4B according to the manufacturer's instructions (Amersham Pharmacia Biotech, Japan). Approximately $4 \mu \mathrm{g}$ of each protein was loaded on a $12 \%$ SDS-polyacrylamide gel.

His-tagged LIM-RFP recombinant proteins were expressed in E. coli and purified following procedures similar to those described above. All primers used in the construction are described in Table $\mathrm{S} 1$.

\section{High- and low-speed cosedimentation assays}

High- and low-speed cosedimentation assays were conducted based on the method as previously described (Han et al., 2013). Rabbit muscle actin proteins were dissolved in A-buffer $\left(5 \mathrm{mmol} \mathrm{L}^{-1}\right.$ Tris- $\mathrm{HCl} \mathrm{pH} 8.0,0.2 \mathrm{mmol} \mathrm{L}^{-1}$ $\mathrm{CaCl}_{2}, 0.2 \mathrm{mmol} \mathrm{L}^{-1} \mathrm{Na}_{2} \mathrm{ATP}$, and $0.5 \mathrm{mmol} \mathrm{L}^{-1}$ dithiothreitol (DTT). All solutions were pre-clarified at $200,000 \times g$ for $1 \mathrm{~h}$. Mg-ATP-actin was prepared by incubation of Ca-ATP-actin on ice with $1 \mathrm{mmol} \mathrm{L}^{-1}$ ethylene glycol tetraacetic acid (EGTA) and $0.1 \mathrm{mmol} \mathrm{L}^{-1} \mathrm{MgCl}_{2}$ for 2 min and was used immediately. Actin $\left(3 \mu \mathrm{mol} \mathrm{L^{-1 }}\right)$ was incubated at $22^{\circ} \mathrm{C}$ for $1 \mathrm{~h}$ either alone or with $3 \mu \mathrm{mol} \mathrm{L}{ }^{-1} \mathrm{LIM}$ proteins in $1 \times$ actin polymerization buffer $(10 \times$ stock: 500 mmol L ${ }^{-1} \mathrm{KCl}, 10 \mathrm{mmol} \mathrm{L}^{-1} \mathrm{MgCl}_{2}, 10 \mathrm{mmol} \mathrm{L}^{-1}$ EGTA,
$100 \mathrm{mmol} \mathrm{L}^{-1}$ imidazole $\mathrm{pH}$ 7.0). The samples were then centrifuged at $200,000 \times g$ for $60 \mathrm{~min}$ in an Optima TLX ultracentrifuge (Beckman, USA) at $4^{\circ} \mathrm{C}$. Proteins in the supernatant and pellets were separated by SDS-PAGE and visualized with Coomassie Brilliant Blue 250 .

In the low-speed cosedimentation assay, WLIM1a and truncated proteins $\left(3 \mu \mathrm{mol} \mathrm{L}{ }^{-1}\right)$ were incubated with $3 \mu \mathrm{mol}$ $\mathrm{L}^{-1}$ preassembled $\mathrm{F}$-actin for $1 \mathrm{~h}$ at $22^{\circ} \mathrm{C}$. After centrifugation at $13,500 \times g$ for $30 \mathrm{~min}$ at $4^{\circ} \mathrm{C}$, samples were analyzed by SDS-PAGE as previously described (Han et al., 2013).

\section{Electrophoretic mobility shift assay (EMSA)}

EMSA was conducted using biotin-labeled probes and the LightShift Chemiluminescent EMSA kit (Pierce, USA). The binding reaction was carried out in a $20 \mu \mathrm{L}$ reaction mixture containing the indicated His-tagged WLIM1a and truncated LIM proteins, $20 \mathrm{nmol} \mathrm{\textrm {L } ^ { - 1 }}$ synthetic biotin-labeled PAL-box DNA probe (ACACCACTAACTCCATCCACTAACTCCAT), and $50 \mathrm{ng} \mu \mathrm{L}^{-1}$ poly (dI-dC). The reaction mixtures were incubated at room temperature for $30 \mathrm{~min}$, and then the samples were separated on a $6 \%$ native polyacrylamide gel in $0.5 \times$ tris-borate-EDTA (TBE) buffer. Electrophoresis was carried out at $15 \mathrm{~V} \mathrm{~cm}^{-1}$ in $0.5 \times \mathrm{TBE}$ buffer. The labeled probes were transferred to a positively charged nylon membrane and were detected using the Chemiluminescent Nucleic Acid Detection Module provided with the kit.

\section{Visualization of actin filaments by fluorescence micros- copy}

F-actin $\left(3 \mu \mathrm{mol} \mathrm{L}{ }^{-1}\right)$ was incubated with LIM-RFPs at room temperature for $30 \mathrm{~min}$ and then labeled with $4 \mu \mathrm{mol} \mathrm{\textrm {L } ^ { - 1 }}$ Alexa Fluor 488 phalloidin (Invitrogen, USA). Actin filaments were subsequently diluted to a final concentration of $10 \mathrm{nmol} \mathrm{\textrm {L } ^ { - 1 }}$ in fluorescence buffer $\left(10 \mathrm{mmol} \mathrm{L}^{-1}\right.$ imidazole pH 7.0, 50 mmol L ${ }^{-1} \mathrm{KCl}, 2 \mathrm{mmol} \mathrm{L}{ }^{-1} \mathrm{MgCl}_{2}, 1 \mathrm{mmol} \mathrm{L}{ }^{-1}$ EGTA, $100 \mathrm{mmol} \mathrm{L}^{-1}$ DTT, $100 \mu \mathrm{g} \mathrm{mL}^{-1}$ Glc oxidase, 15 $\mathrm{mg} \mathrm{mL}^{-1}$ Glc, $20 \mathrm{mg} \mathrm{mL}^{-1}$ catalase, and $0.5 \%$ methylcellulose) as previously described (Han et al., 2013). The diluted samples were visualized using a 1.0 Iris 340 oil immersion lens mounted on an Olympus BX51 microscope. Images were collected with a Leica Spot Pursuit charge-coupled device camera using SPOT version 4.7 software.

\section{In vitro pull-down assay}

Purified His-tagged and GST-tagged LIM proteins were used to perform the pull-down assay. The in vitro pull-down assay was conducted according to the protocol of the ProFound Pull-Down GST Protein Kit (Pierce Biotechnology). The eluted proteins were separated on a SDS-PAGE gel and detected by western blot using anti-GST or anti-His antibodies (1:5,000; Sungene Biotechnology, China). Briefly, 5 $\mu \mathrm{g}$ of GST- or His-tagged WLIM1a and truncated LIM pro- 
teins were used as input and were analyzed by SDS-PAGE and western blot experiments.

\section{Accession numbers}

Sequence data described in this article can be found in the GenBank/EMBL databases under the following accession numbers: WLIM1a (JX648310); AtPLIM2c (NM_115987.3, At3g61230); AtWLIM1 (NM_100894.3, At1g10200); LlLIM1 (ABY21316.1); Ntlim1 (BAA82827); NtWLIM1 (AF184109).

Compliance and ethics The author(s) declare that they have no conflict of interest.

Acknowledgements This work was supported by the National Basic Research Priorities Program (U1303281), and the China Postdoctoral Science Foundation.

Arpat, A.B., Waugh, M., Sullivan, J.P., Gonzales, M., Frisch, D., Main, D., Wood, T., Leslie, A., Wing, R.A., and Wilkins, T.A. (2004). Functional genomics of cell elongation in developing cotton fibers. Plant Mol Biol $54,911-929$.

Cao, X. (2015). Whole genome sequencing of cotton-a new chapter in cotton genomics. Sci China Life Sci 58, 515-516.

Dawid, I.B., Toyama, R., and Taira, M. (1995). Lim domain proteins. $\mathrm{Cr}$ Acad Sci Iii-Vie 318, 295-306.

George, S.P., Wang, Y., Mathew, S., Srinivasan, K., and Khurana, S. (2007). Dimerization and actin-bundling properties of villin and its role in the assembly of epithelial cell brush borders. J Biol Chem 282, 26528-26541

Gou, J., Wang, L., Chen, S., Hu, W., and Chen, X. (2007). Gene expression and metabolite profiles of cotton fiber during cell elongation and secondary cell wall synthesis. Cell Res 17, 422-434.

Graves, D.A., and Stewart, J.M. (1988). Analysis of the protein constituency of developing cotton fibers. J Exp Bot 39, 59-69.

Han, L., Li, Y., Wang, H., Wu, X., Li, C., Luo, M., Wu, S., Kong, Z., Pei, Y., Jiao, G., and Xia, G. (2013). The dual functions of WLIM1a in cell elongation and secondary wall formation in developing cotton fibers. Plant Cell 25, 4421-4438.

Jin, X., Li, Q., Xiao, G., and Zhu, Y. (2013). Using genome- referenced expressed sequence tag assembly to analyze the origin and expression patterns of Gossypium hirsutum transcripts. J Integr Plant Biol 55, 576-585

Kawaoka, A., and Ebinuma, H. (2001). Transcriptional control of lignin biosynthesis by tobacco LIM protein. Phytochemistry 57, 1149-1157.

Kawaoka, A., Kaothien, P., Yoshida, K., Endo, S., Yamada, K., and Ebinuma, H. (2000). Functional analysis of tobacco LIM protein Ntlim1 involved in lignin biosynthesis. Plant J 22, 289-301.

Kim, H.J., and Triplett, B.A. (2001). Cotton fiber growth in planta and in vitro. Models for plant cell elongation and cell wall biogenesis. Plant Physiol 127, 1361-1366.
Li, X., Fan, X., Wang, X., Cai, L., and Yang, W. (2005). The cotton ACTIN1 gene is functionally expressed in fibers and participates in fiber elongation. Plant Cell 17, 859-875.

Moes, D., Gatti, S., Hoffmann, C., Dieterle, M., Moreau, F., Neumann, K., Schumacher, M., Diederich, M., Grill, E., Shen, W.H., Steinmetz, A., and Thomas, C. (2013). A LIM domain protein from tobacco involved in actin-bundling and histone gene transcription. Mol Plant 6, 483-502.

Papuga, J., Hoffmann, C., Dieterle, M., Moes, D., Moreau, F., Tholl, S., Steinmetz, A., and Thomas, C. (2010). Arabidopsis LIM proteins: a family of actin bundlers with distinct expression patterns and modes of regulation. Plant Cell 22, 3034-3052.

Pei, Y. (2015). The homeodomain-containing transcription factor, GhHOX3, is a key regulator of cotton fiber elongation. Sci China Life Sci 58, 309-310.

Ruan, Y., Llewellyn, D.J., and Furbank, R.T. (2003). Suppression of sucrose synthase gene expression represses cotton fiber cell initiation, elongation, and seed development. Plant Cell 15, 952-964.

Shan, C., Shangguan, X., Zhao, B., Zhang, X., Chao, L., Yang, C., Wang, L., Zhu, H., Zeng, Y., Guo, W., Zhou, B., Hu, G., Guan, X., Chen, Z., Wendel, J., Zhang, T., and Chen, X. (2014). Control of cotton fibre elongation by a homeodomain transcription factor GhHOX3. Nat Commun 5, 5519.

Shi, Y., Zhu, S., Mao, X., Feng, J., Qin, Y., Zhang, L., Cheng, J., Wei, L., Wang, Z., and Zhu, Y. (2006). Transcriptome profiling, molecular biological, and physiological studies reveal a major role for ethylene in cotton fiber cell elongation. Plant Cell 18, 651-664.

Thomas, C., Hoffmann, C., Dieterle, M., Van Troys, M., Ampe, C., and Steinmetza, A. (2006). Tobacco WLIM1 is a novel F-actin binding protein involved in actin cytoskeleton remodeling. Plant Cell 18, 2194-2206.

Thomas, C., Moreau, F., Dieterle, M., Hoffmann, C., Gatti, S., Hofmann, C., Van Troys, M., Ampe, C., and Steinmetz, A. (2007). The LIM domains of WLIM1 define a new class of actin bundling modules. J Biol Chem 282, 33599-33608.

Wang, H., Wan, A., and Jauh, G.Y. (2008). An actin-binding protein, L1LIM1, mediates calcium and hydrogen regulation of actin dynamics in pollen tubes. Plant Physiol 147, 1619-1636.

Xu, B., Gou, J., Li, F., Shangguan, X., Zhao, B., Yang, C., Wang, L., Yuan, S., Liu, C., and Chen, X. (2013). A cotton BURP domain protein interacts with-expansin and their co-expression promotes plant growth and fruit production. Mol Plant 6, 945-958.

Xue, X., Guo, C., Du, F., Lu, Q., Zhang, C., and Ren, H. (2011). AtFH8 is involved in root development under effect of low-dose latrunculin B in dividing cells. Mol Plant 4, 264-278.

Zhang, M., Zheng, X., Song, S., Zeng, Q., Hou, L., Li, D., Zhao, J., Wei, Y., Li, X., Luo, M., Xiao, Y., Luo, X., Zhang, J., Xiang, C., and Pei, Y. (2011). Spatiotemporal manipulation of auxin biosynthesis in cotton ovule epidermal cells enhances fiber yield and quality. Nat Biotechnol 29, 453-458.

Zhao, P., Wang, L., Han, L., Wang, J., Yao, Y., Wang, H., Du, X., Luo, Y., and Xia, G. (2010). Proteomic identification of differentially expressed proteins in the Ligon lintless mutant of upland cotton (Gossypium hirsutum L.). J Proteome Res 9, 1076-1087.

Zheng, Q., and Zhao, Y. (2007). The diverse bilofunctions of LIM domain proteins: determined by subcellular localization and protein-protein interaction. Biol Cell 99, 489-502.

Open Access This article is distributed under the terms of the Creative Commons Attribution License which permits any use, distribution, and reproduction in any medium, provided the original author(s) and source are credited.

\section{SUPPORTING INFORMATION}

\section{Table 1 primers used in this study}

The supporting information is available online at life.scichina.com and link.springer.com. The supporting materials are published as submitted, without typesetting or editing. The responsibility for scientific accuracy and content remains entirely with the authors. 